\title{
Coordinated XANES, TEM and SIMS Analysis of the Chemistry, Microstructure and Isotopic Composition of Insoluble Organics in Meteorites
}

\author{
R. M. Stroud, ${ }^{*}$ B. T. De Gregorio, ${ }^{*}$ N. D. Bassim, ${ }^{*}$ T.J. Zega, ${ }^{*}$ L. R. Nittler, ${ }^{*}$ C. M. O’D. \\ Alexander, ${ }^{* *}$ G. D. Cody $^{* *}$ and A. L. D. Kilcoyne** \\ * Materials Science and Technology Division, Naval Research Laboratory, Washington, DC 20375. \\ ** Carnegie Institution of Washington, Washington, DC 20015. \\ *** Advanced Light Source, Lawrence Berkeley National Laboratory, Berkeley, CA 94720.
}

Primordial organic matter, dating to the formation of the Solar System or earlier, can be found in the matrices of primitive chondritic meteorites. The oldest, most primitive organic matter is identified on the basis of large $\mathrm{D}$ and ${ }^{15} \mathrm{~N}$ enrichments compared to typical solar system values. The origin of these isotopic enrichments is not fully known, but may reflect ion-molecular reactions that occurred in the interstellar medium, isotopic compositions inherited from molecules that condensed around stars that predated the Sun, and radiation processing in the interstellar medium or solar nebula. Clues to the formation and processing histories of this material is encoded in the isotopic composition, the $\mathrm{C}, \mathrm{N}$, and $\mathrm{O}$ functional chemistry, and in the physical microstructure. Through coordinated X-ray absorption near edge spectroscopy (XANES), transmission electron microscopy (TEM), and secondary ion mass spectrometry (SIMS) analyses, we seek to better constrain the processes that affected organic matter in the early Solar System.

We characterized insoluble organic matter (IOM) residues produced by acid dissolution of several primitive meteorites [1]. Powdered IOM was embedded in sulfur and ultramicrotomed sections were transferred to TEM grids with $\mathrm{SiO}$ support films. Spherical grains were identified within IOM using a JEOL 2200FS field-emission TEM operated at $200 \mathrm{keV}$ at the Naval Research Laboratory. X-ray absorption near-edge structure spectroscopy (XANES) analyses were performed at beamline 5.3.2 at the Advanced Light Source and at beamline 10-ID1 at the Canadian Light Source. X-ray absorption images were acquired at energies from $275 \mathrm{eV}$ to $420 \mathrm{eV}$. Carbon and nitrogen in various bonding environments absorb photons at discrete energies in this range, allowing mapping of organic functional groups. The $\mathrm{C}$ and $\mathrm{N}$ isotopic composition of the samples were measured with the Carnegie NanoSIMS 50L ion microprobe, operated in multi-detection imaging mode. Because these measurements were performed on thin, ultramicrotomed samples, in many cases secondary ion count rates had not fully stabilized before the support film began to sputter away. Consequently, the measured isotopic enrichments of particular regions are minimum values.

TEM imaging (Fig. 1) of the residues reveals two primary microstructural components, one with a fluffy, microporous texture, and other in the form of solid and hollow spheres, called nanoglobules, that range in size from $\sim 0.1$ to 1 micron. Nearly 100 nanoglobules have been characterized in IOM residues with TEM to date. Although the majority of nanoglobules in an IOM residue from a particular meteorite have similar morphology, there is considerable morphological variability within a given sample. For example, the thickness of nanoglobule walls may vary from $\sim 15 \%$ to nearly $\sim 40 \%$ of the diameter.

Preliminary data [2] indicated that the majority of nanoglobules have XANES spectra that are similar to those of the surrounding microporous IOM, with peak intensity at energies due to photoabsorption from oxygenic functional groups (ketone and carboxyl). We continue to see these 
distinctions in our expanded dataset (blue spectrum in Figure 1C). In addition, about 20\% of nanoglobules from meteorites known to have undergone little aqueous alteration have XANES spectra indicative of highly aromatic organic matter with increased spectral intensity around $285 \mathrm{eV}$ (red spectrum in Figure 1C). Aromatic nanoglobules are rare in meteorites that have been more heavily aqueously altered.

The isotopic compositions of most of the nanoglubules are similar to that of the surrounding IOM. Only around $30 \%$ of the nanoglobules in each meteorite were significantly enriched in ${ }^{15} \mathrm{~N}$ above bulk values. In addition, solid nanoglobules, lacking a hollow core, rarely contain significant ${ }^{15} \mathrm{~N}$ enrichments. However, all but one of the nanoglobules that contained highly aromatic organic matter as measured by XANES were significantly enriched in ${ }^{15} \mathrm{~N}$.

The majority of nanoglobules, which are similar in isotopic composition to typical IOM, are likely derived from aqueous alteration of bulk carbonaceous matter in the host meteorite. These aqueous processes likely solublize amphiphilic material, which often contains oxidized functional groups and can self-assemble into globular structures. Highly aromatic nanoglobules appear to be carriers of largest ${ }^{15} \mathrm{~N}$ isotope enrichments, and therefore most likely to contain presolar organic matter in meteorites. Models of nitrogen isotopic fractionation in cold interstellar environments suggest that

${ }^{15} \mathrm{~N}$ enrichments up to $+4000 \%$ may occur on polycyclic aromatic hydrocarbons (PAHs) [8]. Such enrichments may be preserved by incorporating ${ }^{15} \mathrm{~N}$ into aromatic heterocycles. However, nanoglobules may also be aromatized by radiation exposure within the solar nebula, prior to incorporation into the meteorite parent body.

References

[1] C. M. O’D. Alexander et al. Geochem. Cosmochem. Acta, 71, (2007) 4380.

[2] L. R. Nittler et al. Proceedings of the $40^{\text {th }}$ Lunar and Planetary Science Conference, Abstract \#1145 (2009).
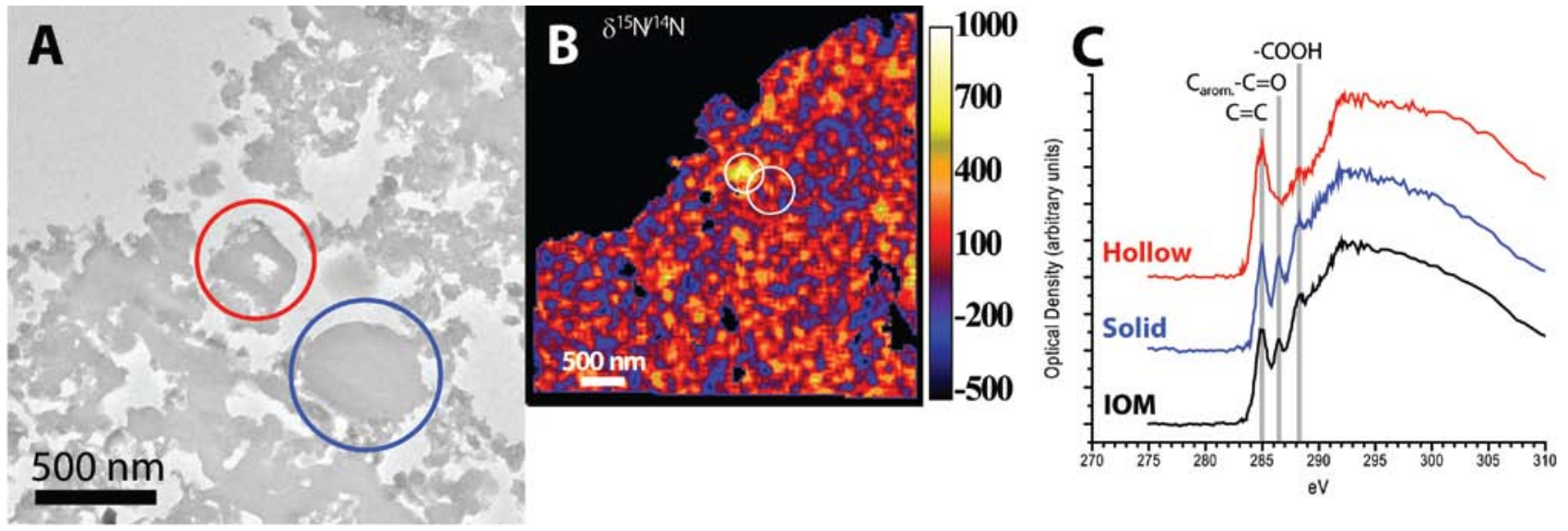

Figure 1. Correlated (A) TEM, (B) SIMS, and (C) XANES characterization of a hollow (red circle) and a solid (blue circle) nanoglobule compared with surrounding insoluble organic matter (IOM) from the meteorite EET92042. 